\title{
MHD Heat and Mass Transfer of Chemical Reaction Fluid Flow over a Moving Vertical Plate in Presence of Heat Source with Convective Surface Boundary Condition
}

\author{
B. R. Rout, ${ }^{1}$ S. K. Parida, ${ }^{2}$ and S. Panda ${ }^{3}$ \\ ${ }^{1}$ Department of Mathematics, Krupajal Engineering College, Prasanti Vihar, Pubasasan, Kausalyaganga, \\ Bhubaneswar, Odisha 751002, India \\ ${ }^{2}$ Department of Mathematics, Institute of Technical Education and Research (ITER), SOA University, \\ Bhubaneswar, Odisha 751019, India \\ ${ }^{3}$ Department of Mathematics and Civil Engineering, National Institute of Technology (NIT) Calicut, Calicut 673601, India
}

Correspondence should be addressed to S. K. Parida; sparidamath2007@rediffmail.com

Received 1 January 2013; Revised 5 March 2013; Accepted 6 March 2013

Academic Editor: Jose C. Merchuk

Copyright (c) 2013 B. R. Rout et al. This is an open access article distributed under the Creative Commons Attribution License, which permits unrestricted use, distribution, and reproduction in any medium, provided the original work is properly cited.

\begin{abstract}
This paper aims to investigate the influence of chemical reaction and the combined effects of internal heat generation and a convective boundary condition on the laminar boundary layer MHD heat and mass transfer flow over a moving vertical flat plate. The lower surface of the plate is in contact with a hot fluid while the stream of cold fluid flows over the upper surface with heat source and chemical reaction. The basic equations governing the flow, heat transfer, and concentration are reduced to a set of ordinary differential equations by using appropriate transformation for variables and solved numerically by Runge-Kutta fourthorder integration scheme in association with shooting method. The effects of physical parameters on the velocity, temperature, and concentration profiles are illustrated graphically. A table recording the values of skin friction, heat transfer, and mass transfer at the plate is also presented. The discussion focuses on the physical interpretation of the results as well as their comparison with previous studies which shows good agreement as a special case of the problem.
\end{abstract}

\section{Introduction}

The study of convective flow with heat and mass transfer under the influence of magnetic field and chemical reaction with heat source has practical applications in many areas of science and engineering. This phenomenon plays an important role in chemical industry, petroleum industry, cooling of nuclear reactors, and packed-bed catalytic reactors. Natural convection flows occur frequently in nature due to temperature differences, concentration differences, and also due to combined effects. The concentration difference may sometimes produce qualitative changes to the rate of heat transfer. The study of heat generation in many fluids due to exothermic and endothermic chemical reactions and natural convection with heat generenation can be added to combustion modeling. In this direction Vajrvelu and Nayfeh [1] studied the hydromagnetic convection at a cone and at a wedge in presence of temperature-dependent heat generation and absorption effect. Chamkha [2] later examined the effect of heat generation or absorption on hydromagnetic threedimensional free convection flow over a vertical stretching surface. The flow through porous media is a subject of most common interest and has emerged as a separate intensive research area because heat and mass transfer in porous medium is very much prevalent in nature and can also be encountered in many technological processes. In this context the effect of temperature-dependent heat sources has been studied by Moalem [3] taking into account the steady state heat transfer within porous medium. Rahman and Sattar [4] have investigated the effect of heat generation or absorption on convective flow of a micropolar fluid past a continuously moving vertical porous plate in presence of a magnetic field. Analysis of transport processes and their interaction with chemical reaction has the greatest contributions to many 
areas of chemical science. The effect of chemical reaction on different geometry of the problem has been investigated by many authors. Das et al. [5] have studied the effect of mass transfer flow past an impulsively started infinite vertical plate with heat flux and chemical reaction. The chemical reaction effect on heat and mass transfer flow along a semiinfinite horizontal plate has been studied by Anjalidevi and Kandaswamy [6] and later it was extended for Hiemenz flow by Seddeek et al. [7] and for polar fluid by Patil and Kulkarni [8]. Salem and Abd El-Aziz [9] have reported the effect of hall currents and chemical reaction on hydromagnetic flow of a stretching vertical surface with internal heat generation or absorption. Ibrahim et al. [10] studied the effect of chemical reaction and radiation absorption on the unsteady MHD free convection flow past a semi-infinite vertical permeable moving plate with heat source and suction. A detailed numerical study has been carried out for unsteady hydromagnetic natural convection heat and mass transfer with chemical reaction over a vertical plate in rotating system with periodic suction by Parida et al. [11]. Rajeswari et al. [12] have investigated chemical reaction, heat and mass transfer on nonlinear MHD boundary layer flow through a vertical porous surface in presence of suction. Mahdy [13] has studied the effect of chemical reaction and heat generation or absorption on double diffussive convection from vertical truncated cone in a porous media with variable viscosity. Pal and Talukdar [14] have studied perturbation analysis of unsteady magnetohydrodynamic convective heat mass transfer in boundary layer slip flow past a vertical permeable plate with a thermal radiation and chemical reaction. Further the effect of thermal radiation, heat and mass transfer flow of a variable viscosity fluid past a vertical porous plate in presence of transverse magnetic field was investigated by Makinde and Ogulu [15]. The analysis of MHD mixedconvection interaction with thermal radiation and higher order chemical reaction is carried out by Makinde [16]. Aziz [17] theoretically examined a similarity solution for a laminar thermal boundary layer over a flat plate with a convective surface boundary condition. He found an interesting result that a similarity solution is possible if the convective heat transfer along with the hot fluid on the lower surface of the plate is inversely proportional to the square root of the axial distance. Recently, the combined effects of an exponentially decaying internal heat generation and a convective boundary condition on the thermal boundary layer over a flat plate are investigated by Olanrewaju et al. [18]. In their study authors have negelected the Sherwood effect. Similar analysis has been carried out by Makinde [19, 20] without heat source and with heat source [21], neglecting chemical reaction effect. There has been considerable interest in studying the effect of chemical reaction [22] and heat source effect on the boundary layer flow problem with heat and mass transfer of an electrically conducting fluid in different geometry [23-25].

Heat source and chemical reaction effects are crucial in controlling the heat and mass transfer. The present paper attempts to investigate the influence of chemical reaction and the combined effects of internal heat generation and the convective boundary condition on the MHD heat and mass transfer flow. To the best of the authors' knowledge, so far no one has considered the combined effect of chemical reaction and heat source along with convective surface boundary condition on MHD flow and the heat and mass transfer over a moving vertical plate. This fact motivated us to propose the similar study. We extend the recent work of Makinde [19], Olanrewaju et al. [18], and Gangadhar et al. [22] to expose the effect of chemical reaction on MHD heat and mass transfer over a moving vertical plate in presence of heat source along with convective surface boundary condition. The coupled nonlinear partial differential equations governing the flow, heat and mass transfer have been reduced to a set of coupled nonlinear ordinary differential equations by using similarity transformation. Following [19] the similarity solutions exist, if the convective heat transfer associated with the hot fluid on lower surface of the plate is proportional to the inverse square root of the axial distance. The reduced equations are solved numerically using Runge-Kutta fourth-order integration scheme together with shooting method. The effect of various physical parameters on the velocity, temperature, and concentration fields is studied.

\section{Mathematical Formulation}

A typical flow scenario is illustrated in Figure 1; it shows a steady two-dimensional boundary layer flow of a stream of cold incompressible electrically conducting fluid over a moving vertical flat plate at temperature $T_{\infty}$ in presence of heat source and chemical reaction. The left surface of the plate is being heated by convection from a hot fluid at temperature $T_{f}$ that gives a heat transfer coefficient $h_{f}$, and $T_{\infty}$ is the temperature of the fluid away from the plate. The cold fluid in contact with the upper surface of the plate generates heat internally at the volumetric rate $Q_{0}$. Here the $x$-axis is taken along the direction of plate and $y$-axis is normal to it. A magnetic field of uniform field strength $B_{0}$ is applied in the negative direction of $y$-axis.

The continuity, momentum, energy, and concentration equations describing the flow under the Boussinesq approximation can be written as

$$
\begin{gathered}
\frac{\partial u}{\partial x}+\frac{\partial v}{\partial y}=0 \\
u \frac{\partial u}{\partial x}+v \frac{\partial u}{\partial y}=v \frac{\partial^{2} u}{\partial y^{2}}-\frac{\sigma B_{0}^{2}}{\rho} u+g \beta\left(T-T_{\infty}\right) \\
+g \beta^{*}\left(C-C_{\infty}\right), \\
u \frac{\partial T}{\partial x}+v \frac{\partial T}{\partial y}=\alpha \frac{\partial^{2} T}{\partial y^{2}}+\frac{Q_{0}}{\rho C_{p}}\left(T-T_{\infty}\right), \\
u \frac{\partial C}{\partial x}+v \frac{\partial C}{\partial y}=D \frac{\partial^{2} C}{\partial y^{2}}-K^{\prime} C .
\end{gathered}
$$

The symbols $u$ and $v$ denote the fluid velocity in the $x$ and $y$-direction. Here $T$ and $C$ are the temperature and concentration variables, $\nu$ is the kinematic viscosity, $\alpha$ is the thermal diffusivity, $D$ is the mass diffusivity, $\beta$ is the thermal expansion coefficient, $\beta^{*}$ is the solutal expansion coefficient, 


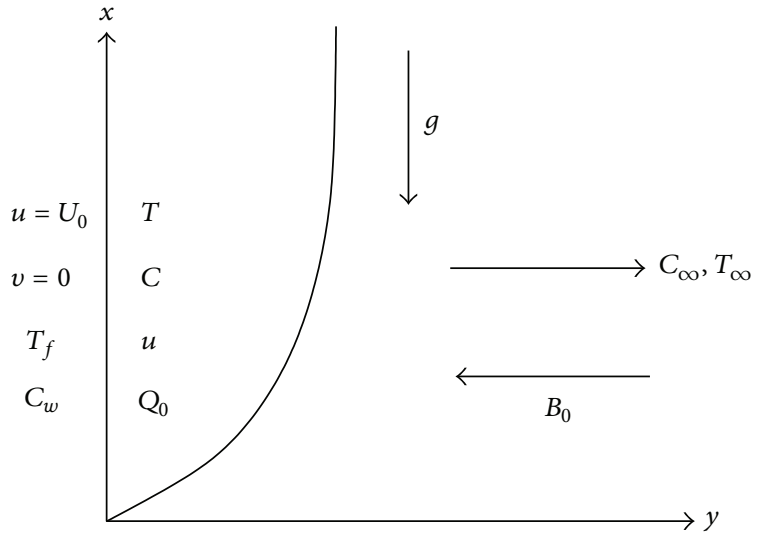

FIGURE 1: Sketch of flow geometry.

$\rho$ is the fluid density, $g$ is the gravitational acceleration, $\sigma$ is the electrical conductivity, $Q_{0}$ is the heat source, $C_{p}$ is the specific heat at constant pressure, and $\mathrm{Kr}^{\prime}$ is the chemical reaction rate on the species concentration. In the above equations, several assumptions have been made. First, the plate is nonconducting, and the effects of radiant heating, viscous dissipation, Hall effects, and induced fields are neglected. Second, the physical properties, that is, viscosity, heat capacity, thermal diffusivity, and the mass diffusivity of the fluid remain invariant throughout the fluid.

The appropriate boundary conditions at the plate surface and far into the cold fluid are

$$
\begin{gathered}
u(x, 0)=U_{0}, \quad v(x, 0)=0, \\
-k \frac{\partial T}{\partial y}(x, 0)=h_{f}\left[T_{f}-T(x, 0)\right], \\
C_{w}(x, 0)=A x^{\lambda}+C_{\infty}, \\
u(x, \infty)=0, \quad T(x, \infty)=T_{\infty}, \quad C(x, \infty)=C_{\infty},
\end{gathered}
$$

where $C_{w}$ is the species concentration at the plate surface, $A$ is the constant, $\lambda$ is the power index of the concentration, $U_{0}$ is the plate velocity, $k$ is the thermal conductivity coefficient, and $C_{\infty}$ is the concentration of the fluid away from the plate. The boundary layer equations presented are nonlinear partial differential equations and, are in general, difficult to solve. However, the equations admit of a self-similar solution. Therefore transformation allows them to be reduced to a system of ordinary differential equations that are relatively easy to solve numerically. We look for solution compatible with (1) of the form

$$
u=U_{0} f^{\prime}(\eta), \quad v=-\frac{1}{2} \sqrt{\frac{\nu U_{0}}{x}} f(\eta)+\frac{U_{0} y}{2 x} f^{\prime}(\eta),
$$

where $\eta=y \sqrt{U_{0} /(\nu x)}$, and prime denotes the differentiation with respect to $\eta$.

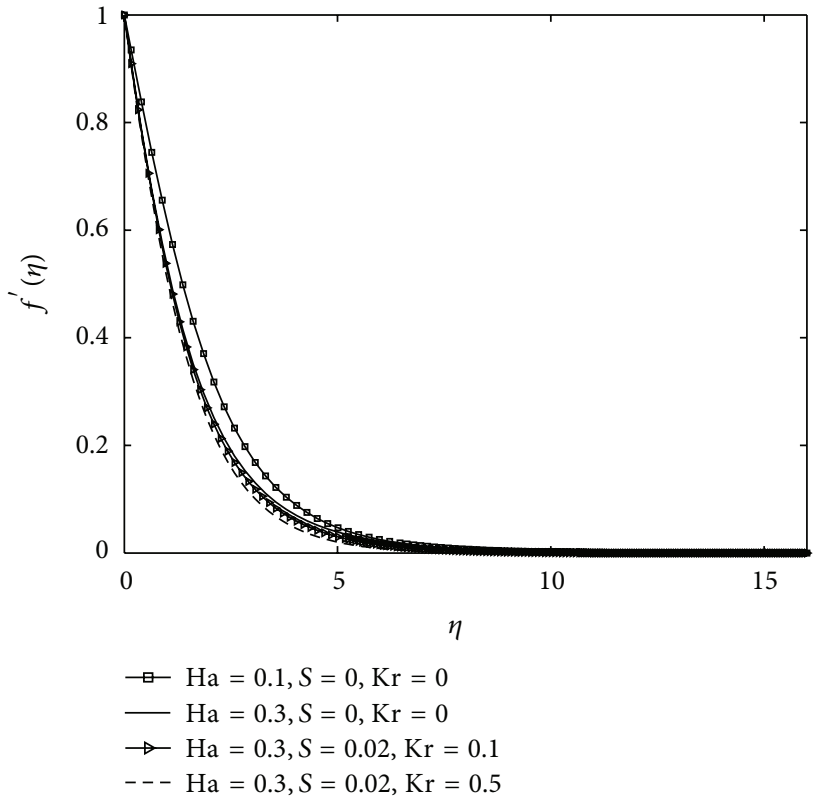

FIGURE 2: Velocity profiles for different values of $\mathrm{Ha}, S$, and $\mathrm{Kr}$ for $\mathrm{Gr}=0.1, \mathrm{Gc}=0.1, \mathrm{Sc}=0.62, \mathrm{Bi}=0.1, \mathrm{Nc}=0.01$, and $\mathrm{Pr}=0.72$.

Let us introduce the dimensionless quantities, that is,

$$
\begin{gathered}
\theta(\eta)=\frac{T-T_{\infty}}{T_{f}-T_{\infty}}, \quad \phi(\eta)=\frac{C-C_{\infty}}{C_{w}-C_{\infty}}, \\
\mathrm{Ha}_{x}=\frac{\sigma B_{0}^{2} x}{\rho U_{0}}, \quad \mathrm{Gr}_{x}=\frac{g \beta\left(T_{f}-T_{\infty}\right) x}{U_{0}^{2}}, \\
\mathrm{Gr}_{x}=\frac{g \beta^{*}\left(C_{w}-C_{\infty}\right) x}{U_{0}^{2}}, \\
\mathrm{Bi}_{x}=\frac{h_{f}}{k} \sqrt{\frac{\nu x}{U_{0}}}, \quad \operatorname{Pr}=\frac{\nu}{\alpha}, \quad \mathrm{Sc}=\frac{\nu}{D}, \\
S_{x}=\frac{Q_{0} x}{U_{0} \rho C_{p}}, \quad \mathrm{Kr}_{x}=\frac{\mathrm{Kr}^{\prime} x}{U_{0}}, \quad \mathrm{Nc}=\frac{C_{\infty}}{C_{w}-C_{\infty}} .
\end{gathered}
$$

Here $\mathrm{Ha}_{x}$ is the local magnetic field parameter, $\mathrm{Gr}_{x}$ is the local thermal Grashof number, $\mathrm{Gc}_{x}$ is the modified Grashof number, $\mathrm{Bi}_{x}$ is the local convective heat transfer parameter, $\mathrm{Pr}$ is the Prandtl number, Sc is the Schmidt number, $S_{x}$ is the local heat source parameter, $\mathrm{Kr}_{x}$ is the local chemical reaction parameter, and $\mathrm{Nc}$ is the concentration difference parameter. Using (6) and (7) in (2)-(4), we get the following equations:

$$
\begin{gathered}
f^{\prime \prime \prime}+\frac{1}{2} f f^{\prime \prime}-\mathrm{Ha}_{x} f^{\prime}+\mathrm{Gr}_{x} \theta+\mathrm{Gc}_{x} \phi=0, \\
\theta^{\prime \prime}+\frac{1}{2} \operatorname{Pr} f \theta^{\prime}+\operatorname{Pr}_{x} \theta=0, \\
\phi^{\prime \prime}+\frac{1}{2} \operatorname{Sc} f \phi^{\prime}-\mathrm{ScKr}_{x}(\phi+\mathrm{Nc})=0 .
\end{gathered}
$$


The corresponding boundary conditions equation (5) for velocity, temperature, and concentration fields in terms of nondimensional variables are

$$
\begin{gathered}
f(0)=0, \quad f^{\prime}(0)=1, \\
\theta^{\prime}(0)=\mathrm{Bi}_{x}[\theta(0)-1], \quad \phi(0)=1, \\
f^{\prime}(\infty)=0, \quad \theta(\infty)=0, \quad \phi(\infty)=0 .
\end{gathered}
$$

It is observed that in the absence of local source parameter and chemical reaction parameter, that is, for $S_{x}=0$ and $\mathrm{Kr}_{x}=0$; (8), (9), and (10) together with boundary condition (11) are the same as those obtained by Makinde [19]. It is noticed that the concentration equation (10) in presence of the chemical reaction parameter $\left(\mathrm{Kr}_{x}\right)$ in the fluid yields nonhomogeneous differential equation which is coupled with momentum equation (8), and in general, difficult to solve analytically. In order to overcome this difficulty, we solve these equations numerically by fourth-order Runge-Kutta method in association with shooting technique. Firstly, these equations together with associated boundary conditions are reduced to first-order differential equations. Since equations to be solved are the third order for the velocity and second order for the temperature and concentration, the values of $f^{\prime}, \theta^{\prime}$, and $\phi^{\prime}$ are needed at $\eta=0$. Therefore, the shooting method is used to solve this boundary value problem. The local skin friction coefficient, the local Nusselt number, the local Sherwood number, and the plate surface temperature are computed in terms of $f^{\prime \prime}(0),-\theta^{\prime}(0),-\phi^{\prime}(0)$ and $\theta(0)$, respectively. It can be noted that the local parameters $\mathrm{Ha}_{x}$, $\mathrm{Gr}_{x}, \mathrm{Gc}_{x}, \mathrm{Bi}_{x}, S_{x}$, and $\mathrm{Kr}_{x}$ in (8)-(10) are functions of $x$ and generate local similarity solution. In order to have a true similarity solution we assume the following relation [19]:

$$
\begin{array}{rlrl}
h_{f}=\frac{a}{\sqrt{x}}, & \sigma=\frac{b}{x}, & \beta & =\frac{c}{x}, \\
\beta^{*}=\frac{d}{x}, & Q_{0}=\frac{e}{x}, & \mathrm{Kr}^{\prime}=\frac{m}{x},
\end{array}
$$

where $a, b, c, d, e$, and $m$ are the constants with appropriate dimensions. In view of relation (12) the parameters $\mathrm{Ha}_{x}$, $\mathrm{Gr}_{x}, \mathrm{Gc}_{x}, \mathrm{Bi}_{x}, S_{x}$, and $\mathrm{Kr}_{x}$ are now independent of $x$ and henceforth, we drop the index " $x$ " for simplicity.

\section{Result Discussion}

The numerical solutions of the boundary value problem for system of ordinary differential equations were obtained by Runge-Kutta method along with shooting technique. Since the physical domain of the underlying problem is unbounded, the computational domain is chosen sufficiently large in order to meet the far field boundary condition at infinity. Here the transverse distance is fixed to 10 and suitably more than 10 depending upon the choice of the parameters. To demonstrate successful implementation of the numerical scheme, the numerical results are compared to those obtained by a previous published paper (see [19]) for the local skin friction coefficient, plate surface temperature, and the local

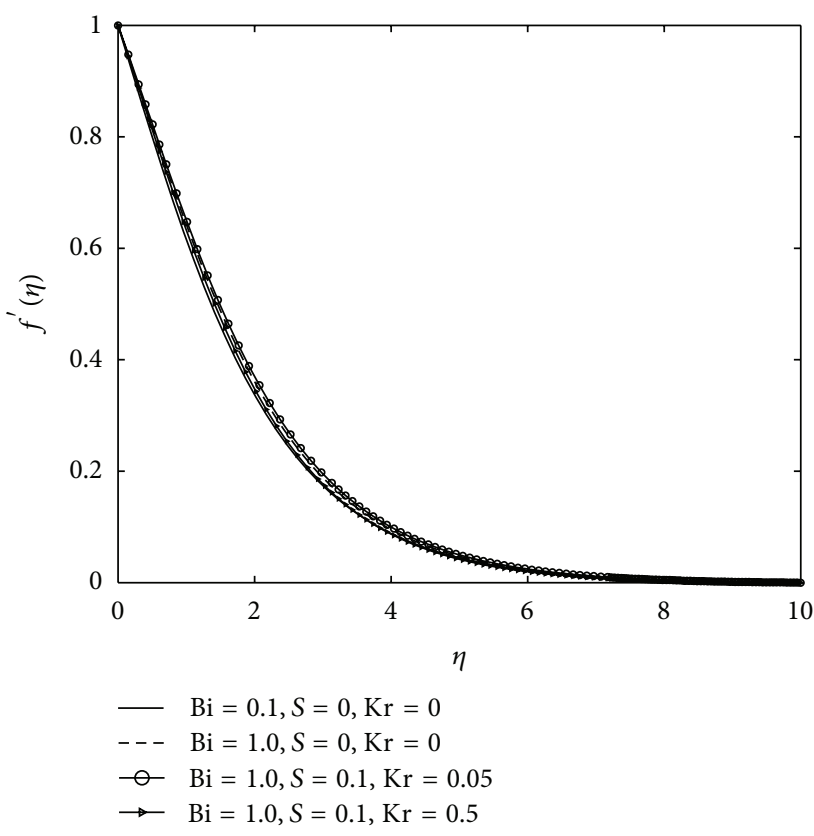

FIGURE 3: Velocity profiles for different values of $\mathrm{Bi}, S$, and $\mathrm{Kr}$ for $\mathrm{Ha}=0.1, \mathrm{Gr}=0.1, \mathrm{Gc}=0.1, \mathrm{Sc}=0.62, \mathrm{Nc}=0.01$, and $\operatorname{Pr}=0.72$.

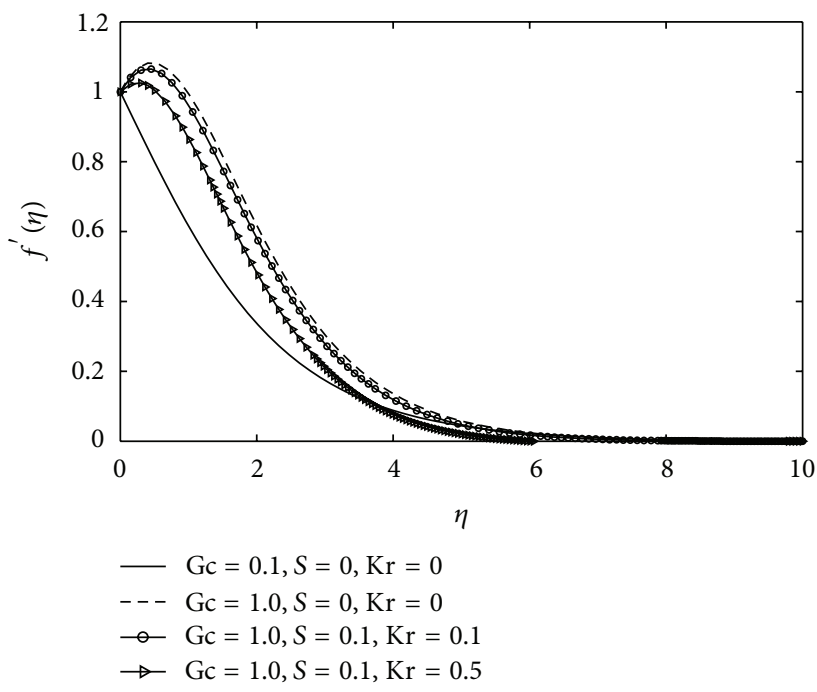

Figure 4: Velocity profiles for different values of $\mathrm{Gc}, S$, and $\mathrm{Kr}$ for $\mathrm{Ha}=0.1, \mathrm{Gr}=0.1, \mathrm{Sc}=0.62, \mathrm{Bi}=0.1, \mathrm{Nc}=0.01$, and $\mathrm{Pr}=0.72$.

Sherwood and Nusselt numbers in Table 1 for the parameters embedded in absence of local chemical reaction parameter $(\mathrm{Kr})$ and local heat source parameter $(S)$. Table 1 presents a comparison of $f^{\prime \prime}(0),-\theta^{\prime}(0), \theta(0)$, and $-\phi^{\prime}(0)$ between the present results and the results obtained by Makinde [19] for various values of $\mathrm{Ha}, \mathrm{Gr}, \mathrm{Gc}, \mathrm{Bi}, \mathrm{Pr}$, and $\mathrm{Sc}$ when $S=\mathrm{Kr}=$ 0 . The results are found to be in excellent agreement. It is important to note that the momentum equation is coupled with heat and mass transfer equations and hence the Prandtl number, Schmidt number, chemical reaction parameter, and source term have an influence on skin friction in our present 
TABle 1: Comparison of $f^{\prime \prime}(0),-\theta^{\prime}(0), \theta(0)$, and $-\phi^{\prime}(0)$ for various values of $\mathrm{Bi}, \mathrm{Ha}, \mathrm{Gr}, \mathrm{Gc}, \mathrm{Pr}$, and Sc when $\mathrm{Kr}=S=0$.

\begin{tabular}{ccccccccccccccc}
\hline Bi & Gr & Gc & Ha & Pr & Sc & \multicolumn{9}{c}{ Makinde $[19]$} \\
& & & & & & $f^{\prime \prime}(0)$ & $-\theta^{\prime}(0)$ & $\theta(0)$ & $-\phi^{\prime}(0)$ & $f^{\prime \prime}(0)$ & $-\theta^{\prime}(0)$ & $\theta(0)$ & $-\phi^{\prime}(0)$ \\
\hline 0.1 & 0.1 & 0.1 & 0.1 & 0.72 & 0.62 & -0.402271 & 0.078635 & 0.213643 & 0.3337425 & -0.402271 & 0.078635 & 0.213643 & 0.333742 \\
1.0 & 0.1 & 0.1 & 0.1 & 0.72 & 0.62 & -0.352136 & 0.273153 & 0.726846 & 0.3410294 & -0.352136 & 0.273153 & 0.726846 & 0.341029 \\
0.1 & 0.5 & 0.1 & 0.1 & 0.72 & 0.62 & -0.322212 & 0.079173 & 0.208264 & 0.3451301 & -0.322212 & 0.079173 & 0.208264 & 0.345130 \\
0.1 & 1.0 & 0.1 & 0.1 & 0.72 & 0.62 & -0.231251 & 0.079691 & 0.203088 & 0.3566654 & -0.231251 & 0.079691 & 0.203088 & 0.356665 \\
0.1 & 0.1 & 0.5 & 0.1 & 0.72 & 0.62 & -0.026410 & 0.080711 & 0.192889 & 0.3813954 & -0.026410 & 0.080711 & 0.192889 & 0.381395 \\
0.1 & 0.1 & 1.0 & 0.1 & 0.72 & 0.62 & 0.3799184 & 0.082040 & 0.179592 & 0.4176699 & 0.379918 & 0.082040 & 0.179592 & 0.417669 \\
0.1 & 0.1 & 0.1 & 5.0 & 0.72 & 0.62 & -2.217928 & 0.066156 & 0.338435 & 0.1806634 & -2.217928 & 0.066156 & 0.338435 & 0.180664 \\
0.1 & 0.1 & 0.1 & 0.1 & 1.0 & 0.62 & -0.407908 & 0.081935 & 0.180640 & 0.3325180 & -0.407908 & 0.081935 & 0.180640 & 0.332518 \\
0.1 & 0.1 & 0.1 & 0.1 & 7.10 & 0.62 & -0.421228 & 0.093348 & 0.066513 & 0.3305618 & -0.421431 & 0.093348 & 0.066515 & 0.330843 \\
0.1 & 0.1 & 0.1 & 0.1 & 0.72 & 0.78 & -0.411704 & 0.078484 & 0.215159 & 0.3844559 & -0.411704 & 0.078484 & 0.215159 & 0.384455 \\
0.1 & 0.1 & 0.1 & 0.1 & 0.72 & 2.63 & -0.453094 & 0.077915 & 0.220841 & 0.7981454 & -0.453094 & 0.077915 & 0.220841 & 0.798146 \\
\hline
\end{tabular}

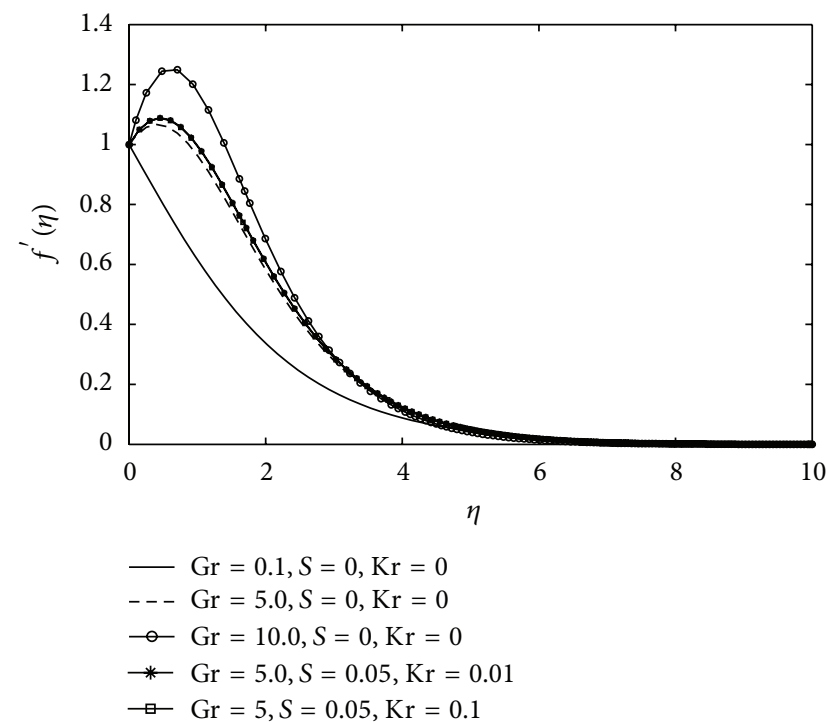

FIGURE 5: Velocity profiles for different values of $\mathrm{Gr}, S$, and $\mathrm{Kr}$ for $\mathrm{Ha}=0.1, \mathrm{Gc}=0.1, \mathrm{Sc}=0.62, \mathrm{Bi}=0.1, \mathrm{Nc}=0.01$, and $\mathrm{Pr}=0.72$.

problem. The influence of heat source and chemical reaction parameters on local skin friction, local Nusselt number, plate surface temperature, and Sherwood number are highlighted in Tables 2 and 3 for various nondimensional flow parameters. It is clearly seen from Table 2 that the magnitude of skin friction and Nusselt number increase whereas the plate surface temperature, and Sherwood number decrease with the increase of source parameter. Furthermore, increasing the strength of chemical reacting substances is to increase the local skin friction, the plate surface temperature and Sherwood number but opposite behavior is seen for local Nusselt number. The obvious observation from Table 2 is that the fluid low Prandtl number increases the magnitude of local skin friction and local Nusselt number while decreasing the plate surface temperature and local Sherwood number. Again the fluid with low convective resistance (or external resistance) decreases the magnitude of local skin friction while the Nusselt number, the plate surface temperature, and local Sherwood number increase. It is also observed

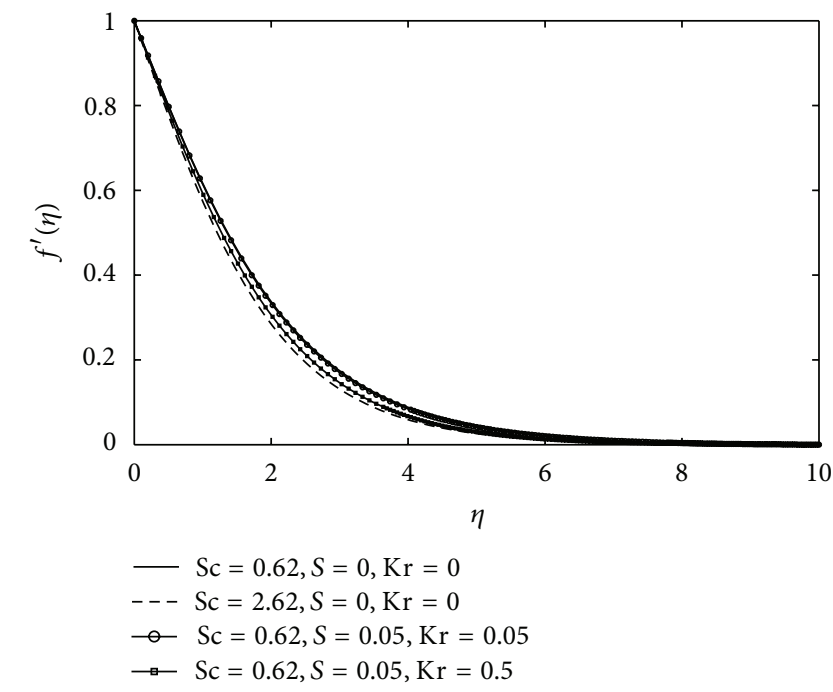

FIGURE 6: Velocity profiles for different values of Sc, $S$, and $\mathrm{Kr}$ for $\mathrm{Ha}=0.1, \mathrm{Gr}=0.1, \mathrm{Gc}=0.1, \mathrm{Bi}=0.1, \mathrm{Nc}=0.01$, and $\mathrm{Pr}=0.72$.

TABLE 2: Computation of skin friction $\left(f^{\prime \prime}(0)\right)$, Nusselt number $\left(-\theta^{\prime}(0)\right)$, plate surface temperature $(\theta(0))$, and Sherwood number $\left(-\phi^{\prime}(0)\right)$ for different values of $\mathrm{Bi}, \mathrm{Pr}, S$, and $\mathrm{Kr}$. The other parameters are $\mathrm{Ha}=0.1, \mathrm{Gr}=0.1, \mathrm{Gc}=0.1, \mathrm{Sc}=0.62$, and $\mathrm{Nc}=0.01$.

\begin{tabular}{cccccccc}
\hline $\mathrm{Bi}$ & $\mathrm{Pr}$ & $S$ & $\mathrm{Kr}$ & $f^{\prime \prime}(0)$ & $-\theta^{\prime}(0)$ & $\theta(0)$ & $-\phi^{\prime}(0)$ \\
\hline 0.1 & 0.72 & 0.1 & 0.1 & -0.604781 & 0.154830 & 0.548301 & 0.376062 \\
0.5 & 0.72 & 0.1 & 0.1 & -0.358517 & 0.167824 & 0.664351 & 0.428429 \\
1.0 & 0.72 & 0.1 & 0.1 & -0.344220 & 0.204287 & 0.795712 & 0.430013 \\
1.0 & 0.72 & 0.3 & 0.1 & -0.410247 & 0.283270 & 0.716729 & 0.413375 \\
1.0 & 0.72 & 0.3 & 0.3 & -0.419822 & 0.265179 & 0.734820 & 0.552735 \\
1.0 & 0.72 & 0.3 & 0.6 & -0.428749 & 0.253741 & 0.746258 & 0.708364 \\
1.0 & 1.0 & 0.3 & 0.6 & -0.412617 & 0.225282 & 0.774717 & 0.709739 \\
\hline
\end{tabular}

from Table 3 that the increase of Schimdt number results in increase of the magnitude of the local skin friction but opposite behavior is marked in case of Nusselt number and the plate surface temperature. 
TABle 3: Computation of skin friction $\left(f^{\prime \prime}(0)\right)$, Nusselt number $\left(-\theta^{\prime}(0)\right)$, plate surface temperature $(\theta(0))$, and Sherwood number $\left(-\phi^{\prime}(0)\right)$ for different values of Sc, $S$, and $\mathrm{Kr}$. The other parameters are fixed at $\mathrm{Bi}=\mathrm{Gc}=\mathrm{Gr}=0.1, \mathrm{Pr}=0.72$, and $\mathrm{Nc}=0.01$.

\begin{tabular}{lcccccc}
\hline Sc & $S$ & $\mathrm{Kr}$ & $f^{\prime \prime}(0)$ & $-\theta^{\prime}(0)$ & $\theta(0)$ & $-\phi^{\prime}(0)$ \\
\hline 0.62 & 0.05 & 0.05 & -0.403557 & 0.075603 & 0.243969 & 0.381077 \\
0.62 & 0.1 & 0.05 & -0.395738 & 0.070777 & 0.292201 & 0.382425 \\
0.62 & 0.3 & 0.05 & -0.433542 & 0.087237 & 0.127620 & 0.373824 \\
0.62 & 0.1 & 0.5 & -0.606262 & 0.141007 & -0.410071 & 0.648094 \\
0.62 & 0.1 & 1.0 & -0.612201 & 0.137188 & -0.371886 & 0.864236 \\
0.78 & 0.1 & 1.0 & -0.615507 & 0.135984 & -0.359841 & 0.972618 \\
2.63 & 0.1 & 1.0 & -0.634123 & 0.132985 & -0.329852 & 1.811414 \\
\hline
\end{tabular}

3.1. Velocity Profiles. Figures 2-7 exhibit the velocity profiles obtained by the numerical simulations for various flow parameters involved in the problem. The simulated parameters are reported in the figure caption. The effects of magnetic parameter on the velocity field in presence and absence of source and chemical reaction parameter are shown in Figure 2. It illustrates that the velocity profile decreases with the increase of magnetic parameter in absence of source and chemical reaction parameter because Lorentz force acts against the flow if the magnetic field is applied in the normal direction. In presence of source and chemical reaction parameter no such appreciable change is observed in Figure 2. This corresponds to Figure (2) in [19] and thereby again validating our numerical scheme. A little increase in the velocity profile near the boundary layer is marked in Figure 3 with the increase in the convective heat parameter because the fluid adjacent to the right surface of the plate becomes lighter by hot fluid and rises faster. The boundary layer flows develop adjacent to vertical surface and velocity reaches a maximum in the boundary layer. It is evident from Figures 4 and 5 that greater cooling of surface, an increase in Gc, and increase in Gr result in an increase in the velocity. It is due to the fact that the increase in the values of Grashof number and modified Grashof number has the tendency to increase the thermal and mass buoyancy effect. The increase is also evident due to the presence of source and chemical reaction parameters. Furthermore the velocity increases rapidly and suddenly falls near the boundary and then approaches the far field boundary condition due to favorable buoyancy force with the increase of both Gr and Gc. It can be seen that the increase in the Prandtl number and Schmidt number leads to a fall in the velocity as shown in Figures 6 and 7 .

3.2. Temperature Profiles. Figures $8-13$ show the temperature profiles obtained by the numerical simulations for various values of flow parameters. Figure 8 clearly demonstrates that the temperature profiles increase with the increase of the magnetic field parameter, which implies that the applied magnetic field tends to heat the fluid, and thus reduces the heat transfer from the wall. Further it can be seen that temperature profile increases due to increase of heat source as well as chemical reaction parameter. The thermal boundary layer thickness increases with an increase in the

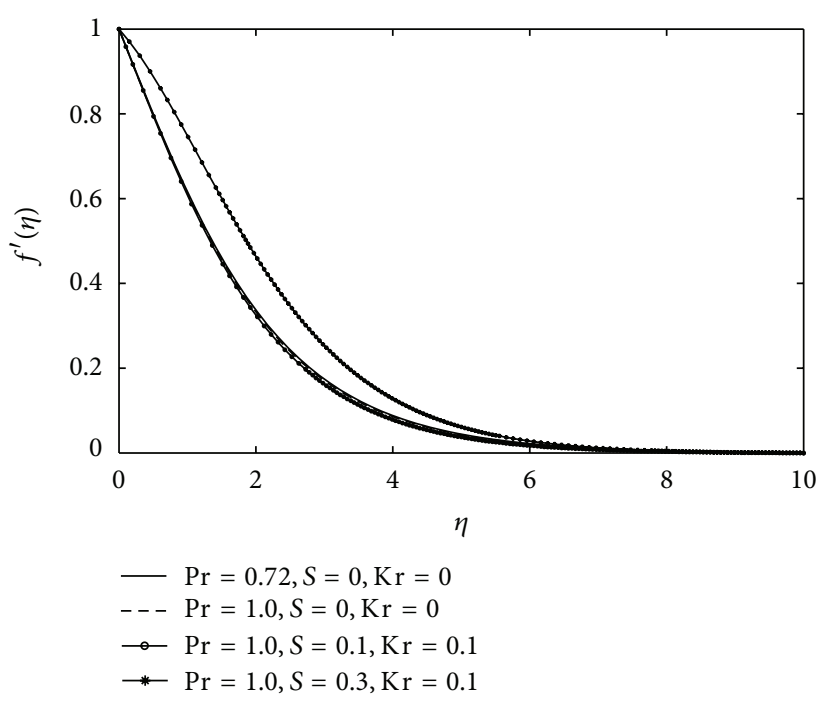

Figure 7: Velocity profiles for different values of $\mathrm{Pr}, S$, and $\mathrm{Kr}$ for $\mathrm{Ha}=0.1, \mathrm{Gr}=0.1, \mathrm{Gc}=0.1, \mathrm{Sc}=0.62, \mathrm{Bi}=0.1$, and $\mathrm{Nc}=0.01$.

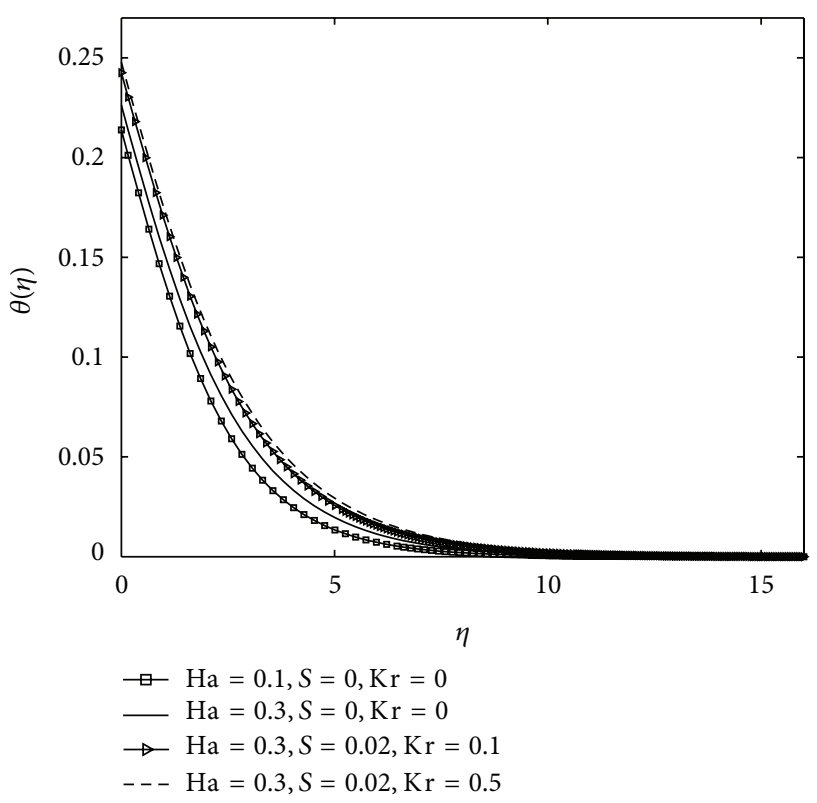

FIgURE 8: Temperature profiles for different values of $\mathrm{Ha}, S$, and $\mathrm{Kr}$ for $\mathrm{Gr}=0.1, \mathrm{Gc}=0.1, \mathrm{Sc}=0.62, \mathrm{Bi}=0.1, \mathrm{Nc}=0.01$, and $\mathrm{Pr}=0.72$.

plate surface convective heat parameter (Figure 9), and a similar effect is also observed in Figure 12 with the increase of Schmidt number. It can be observed that the amplitude of fluid temperature in presence of heat source and chemical reacting substances is more in comparison to in absence of these parameters. The steady state temperatures for different Grashof number, modified Grashof number, internal heat source, and chemical reaction parameters are shown in Figures 10 and 11. The thermal boundary layer decreases with increasing Grashof number and modified Grashof number, but reverse effect is observed with the presence of chemical reaction parameter. This is also revealed in Figure 13, which 


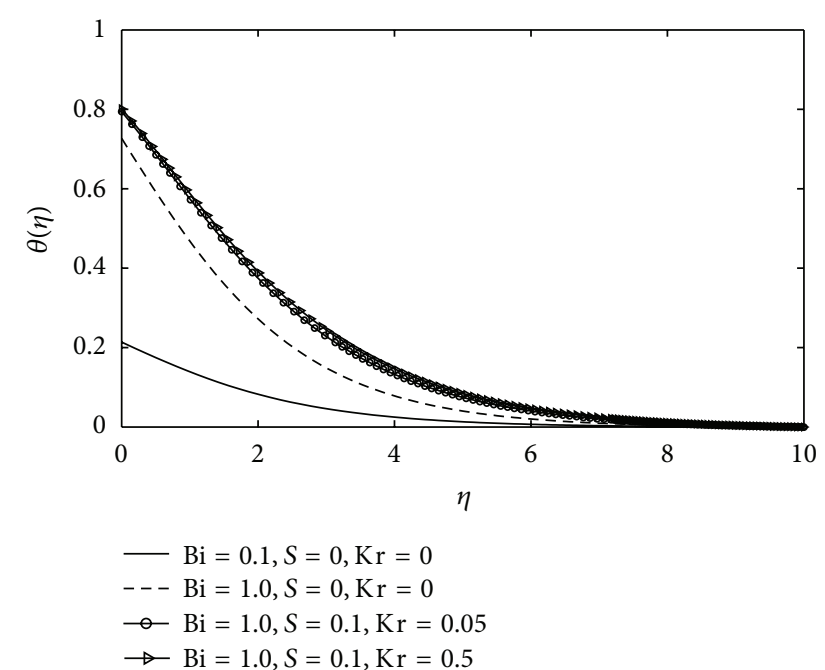

FIGURE 9: Temperature profiles for different values of $\mathrm{Bi}, S$, and $\mathrm{Kr}$ for $\mathrm{Ha}=0.1, \mathrm{Gr}=0.1, \mathrm{Gc}=0.1, \mathrm{Sc}=0.62, \mathrm{Nc}=0.01$, and $\mathrm{Pr}=$ 0.72 .

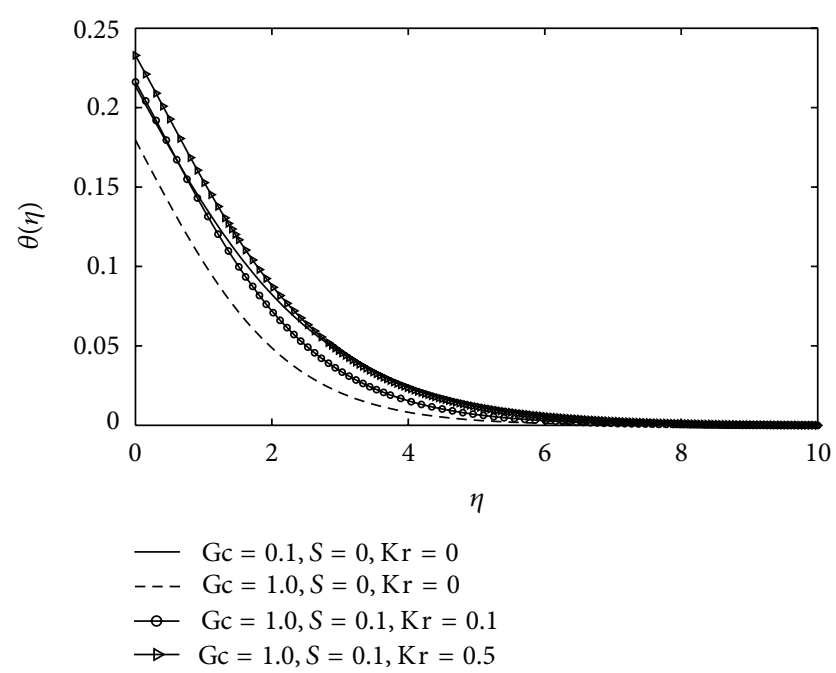

Figure 10: Temperature profiles for different values of Gc, $S$, and $\mathrm{Kr}$ for $\mathrm{Ha}=0.1, \mathrm{Gr}=0.1, \mathrm{Sc}=0.62, \mathrm{Bi}=0.1, \mathrm{Nc}=0.01$, and $\mathrm{Pr}=0.72$.

shows that thermal boundary layer thickness decreases as the Prandtl number increases implying higher heat transfer. It is due to that smaller values of Pr means increasing the thermal conductivity, and therefore heat is able to diffuse away from the plate more quickly than higher values of $\mathrm{Pr}$, hence the rate of heat transfer is reduced. It is noted that, owing to the presence of heat source effect $(S>0)$ and chemical reaction parameter $(\mathrm{Kr}>0)$, the thermal state of the fluid increases. Hence, the temperature of the fluid increases within the boundary layers. In the event that the strength of the heat source and chemical reaction parameters are relatively large, a remarkable change is observed in the temperature profiles within the thermal boundary layer as can be seen in Figure 13. Further, the effect of heat generation

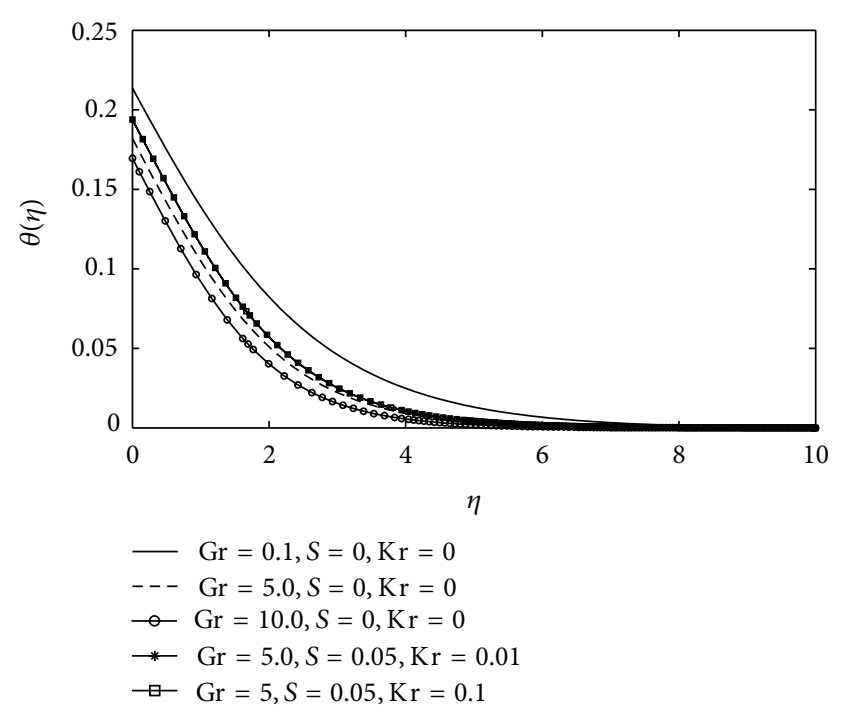

FIgURE 11: Temperature profiles for different values of $\mathrm{Gr}, S$, and $\mathrm{Kr}$ for $\mathrm{Ha}=0.1, \mathrm{Gc}=0.1, \mathrm{Sc}=0.62, \mathrm{Bi}=0.1, \mathrm{Nc}=0.01$, and $\mathrm{Pr}=0.72$.

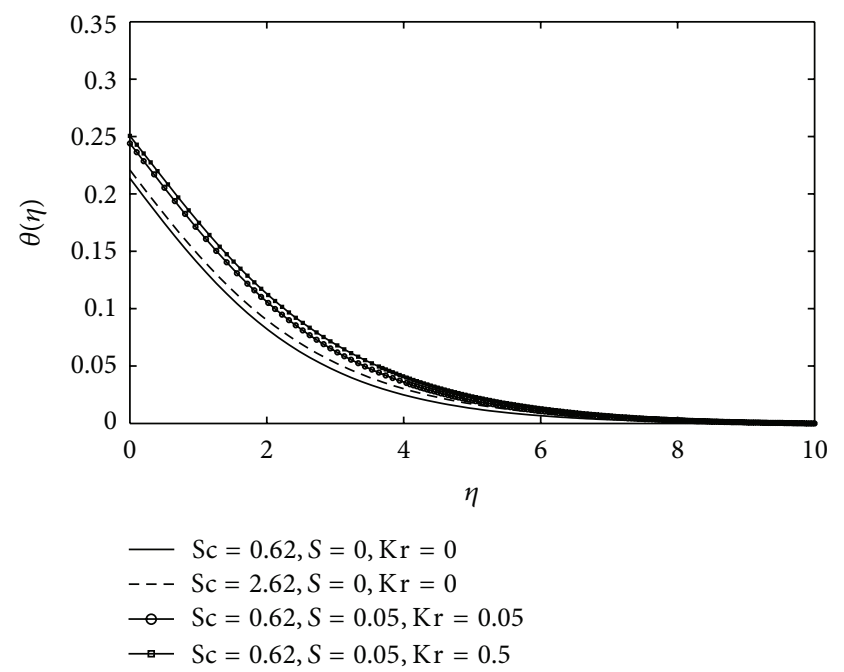

FIGURE 12: Temperature profiles for different values of Sc, $S$, and $\mathrm{Kr}$ for $\mathrm{Ha}=0.1, \mathrm{Gr}=0.1, \mathrm{Gc}=0.1, \mathrm{Bi}=0.1, \mathrm{Nc}=0.01$, and $\mathrm{Pr}=0.72$.

is more pronounced on temperature profiles for high Prandtl number fluids.

3.3. Concentration Profile. Figures $14-19$ show the concentration profiles obtained by the numerical simulations for various values of nondimensional parameters $\mathrm{Bi}, \mathrm{Ha}, \mathrm{Gr}$, Gc, $\mathrm{Kr}, \mathrm{Pr}, \mathrm{Nc}, \mathrm{Sc}$, and $S$. In Figure 14, the effect of an applied magnetic field is found to increase the concentration boundary layer. However, it is interesting to note that the concentration profiles decrease with the increase of both heat source and chemical reaction parameters (Figures 14 and 15). Figures 16 and 17 reveal the concentration variations with Gr, and $\mathrm{Gc}$ respectively. It is due to the fact that an increase in the values of Grashof number and modified Grashof number has the tendency to increase the mass buoyancy effect. This gives 


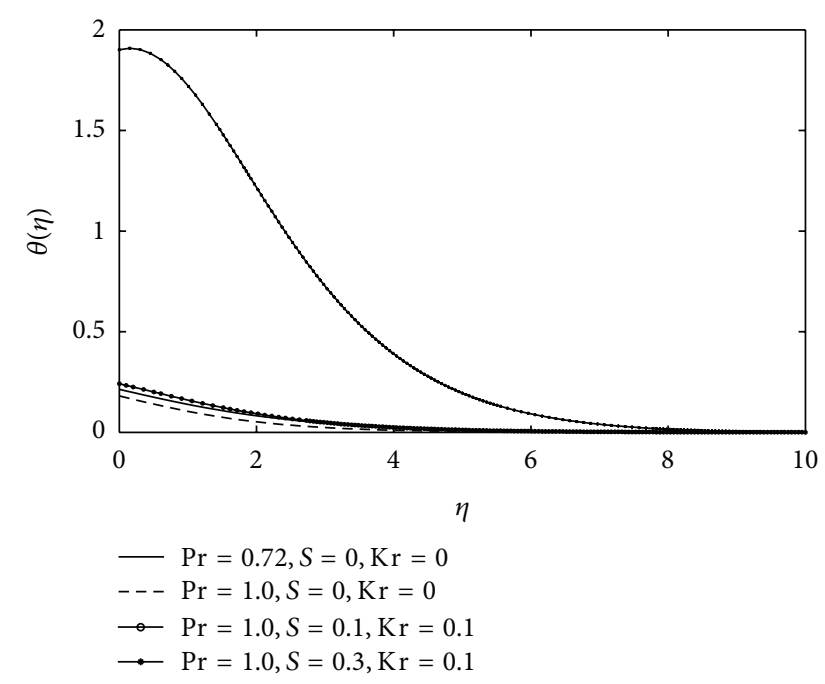

FIGURE 13: Temperature profiles for different values of $\mathrm{Pr}, S$, and $\mathrm{Kr}$ for $\mathrm{Ha}=0.1, \mathrm{Gr}=0.1, \mathrm{Gc}=0.1, \mathrm{Sc}=0.62, \mathrm{Bi}=0.1$, and $\mathrm{Nc}=0.01$.

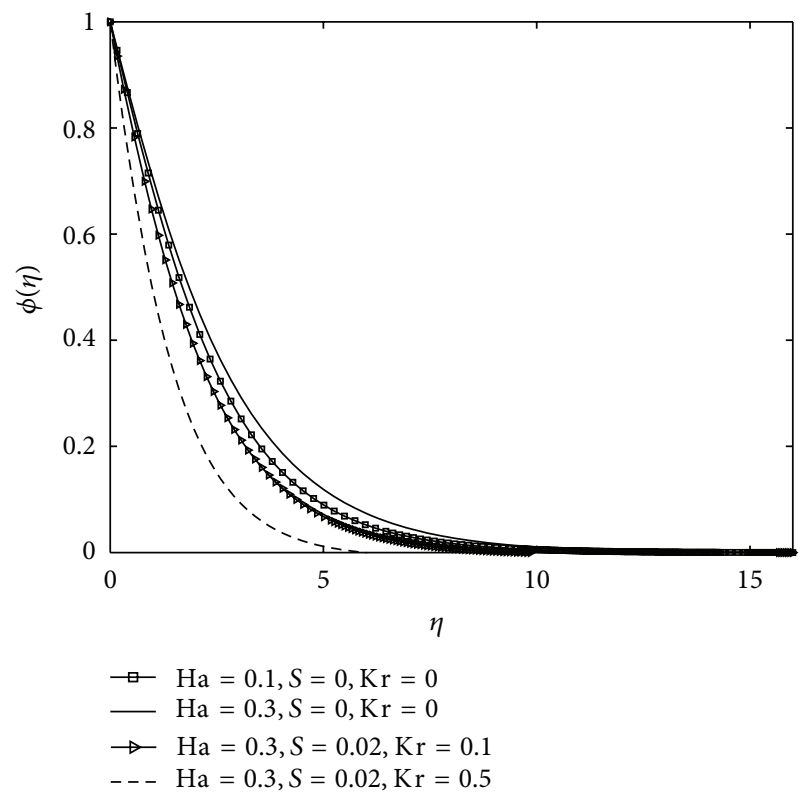

FIGURE 14: Concentration profiles for different values of $\mathrm{Ha}, S$, and $\mathrm{Kr}$ for $\mathrm{Gr}=0.1, \mathrm{Gc}=0.1, \mathrm{Sc}=0.62, \mathrm{Bi}=0.1, \mathrm{Nc}=0.01$, and $\operatorname{Pr}=0.72$.

rise to an increase in the induced flow and thereby decreases concentration. Figure 18 depicts the effect of Schmidt number on the concentration. Like temperature, the concentration value is higher at the surface and falls exponentially. The concentration decreases with an increase in Sc and the decrease is more with the increase in concentration parameter. Figure 19 displays the effect of Pr on concentration profile against $\eta$ with the variation of source and chemical reaction parameters. The magnitude of concentration is higher at the plate and then decays to zero asymptotically; this is due to the fact that thermal conductivity of fluid decreases with the increase of $\mathrm{Pr}$

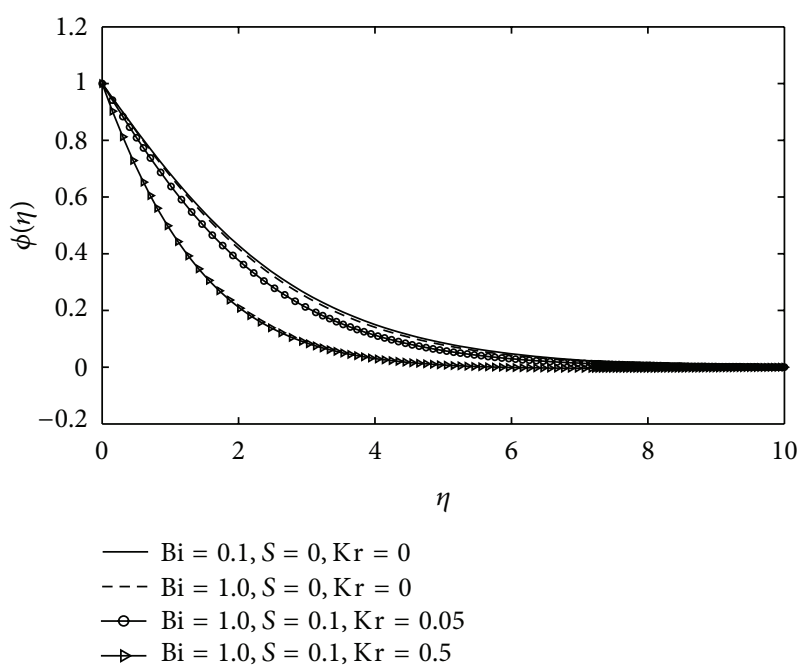

FIgURE 15: Concentration profiles for different values of $\mathrm{Bi}, S$, and $\mathrm{Kr}$ for $\mathrm{Ha}=0.1, \mathrm{Gr}=0.1, \mathrm{Gc}=0.1, \mathrm{Sc}=0.62, \mathrm{Nc}=0.01$, and $\operatorname{Pr}=0.72$.

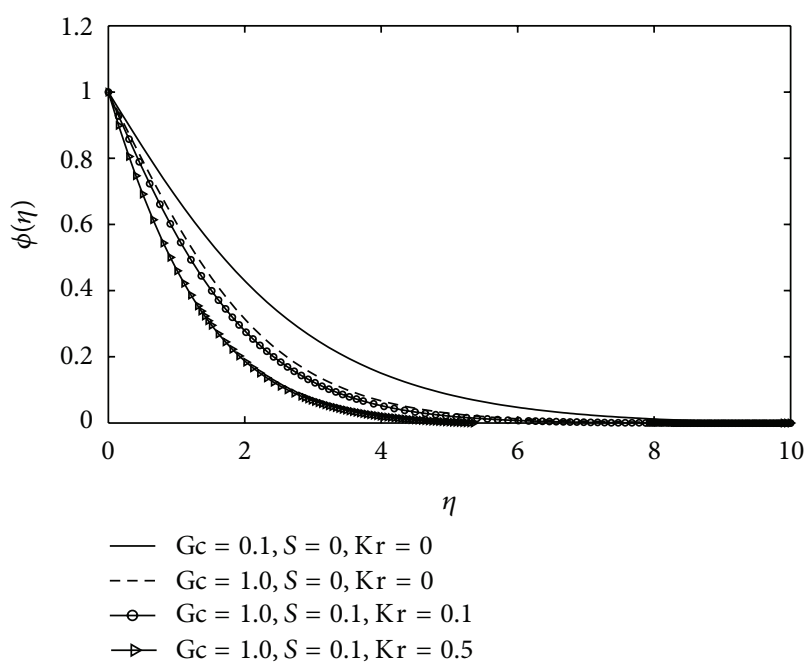

FIGURE 16: Concentration profiles for different values of Gc, $S$, and $\mathrm{Kr}$ for $\mathrm{Ha}=0.1, \mathrm{Gr}=0.1, \mathrm{Sc}=0.62, \mathrm{Bi}=0.1, \mathrm{Nc}=0.01$, and $\operatorname{Pr}=0.72$.

resulting a decrease in thermal boundary layer thickness, and source term further influences the decrease of concentration.

\section{Conclusions}

The present numerical study has been carried out for heat and mass transfer of MHD flow over a moving vertical plate in presence of heat source and chemical reaction along with convective surface boundary condition. The shooting method with Runge-Kutta fourth-order iteration scheme has been implemented to solve the dimensionless velocity, thermal, and mass boundary layer equations. It has been shown that the magnitude of local skin friction and local Nusselt number increase whereas the plate surface temperature and 


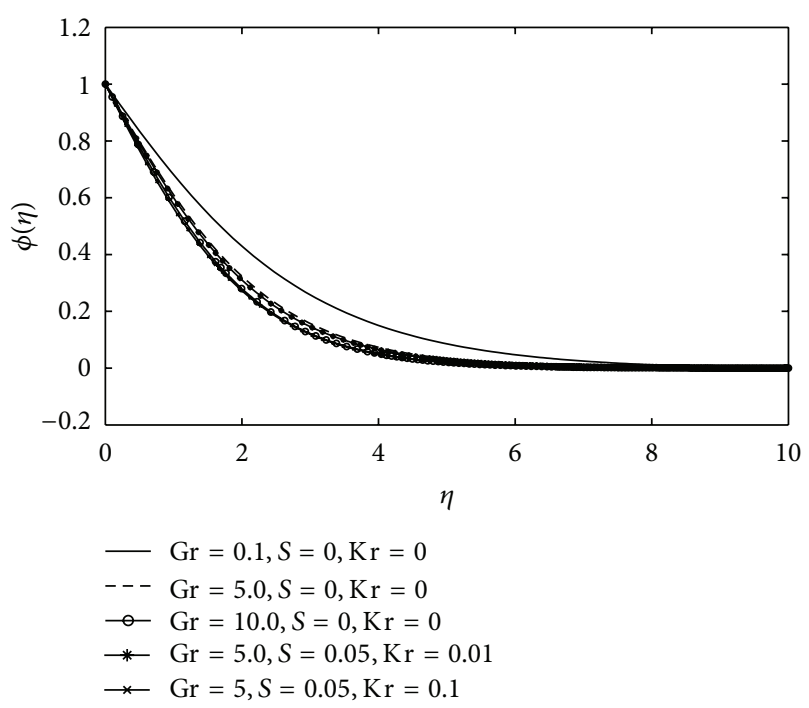

FIGURE 17: Concentration profiles for different values of Gr, $S$, and $\mathrm{Kr}$ for $\mathrm{Ha}=0.1, \mathrm{Gc}=0.1, \mathrm{Sc}=0.62, \mathrm{Bi}=0.1, \mathrm{Nc}=0.01$, and $\operatorname{Pr}=0.72$.

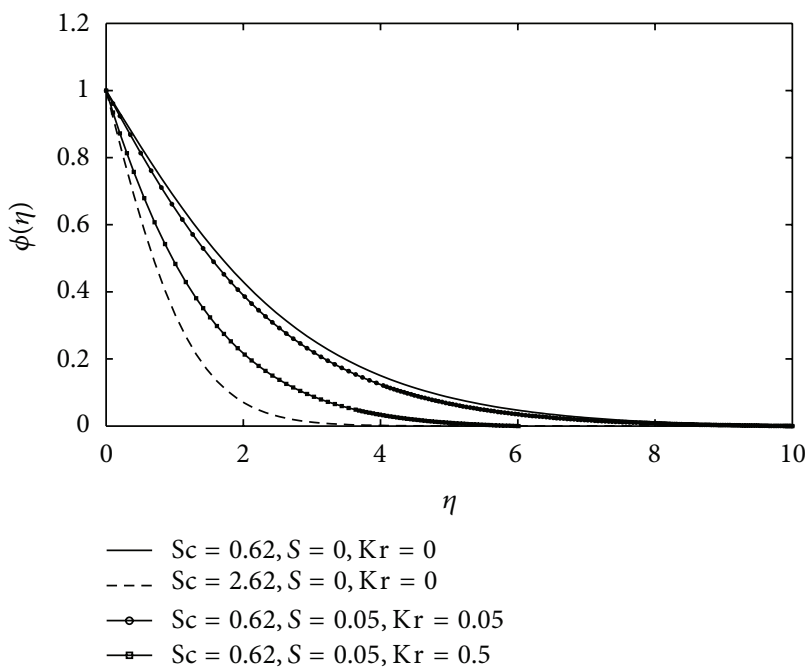

Figure 18: Concentration profiles for different values of Sc, $S$, and $\mathrm{Kr}$ for $\mathrm{Ha}=0.1, \mathrm{Gr}=0.1, \mathrm{Gc}=0.1, \mathrm{Bi}=0.1, \mathrm{Nc}=0.01$, and $\operatorname{Pr}=0.72$.

Sherwood number decreases with an increase in source parameter. The increase in the strength of chemical reacting substances causes an increase in the magnitude of local skin friction, the plate surface temperature, and Sherwood number, but opposite behavior is seen for local Nusselt number. The velocity profile decreases by increasing the magnetic parameter and even the increase is more prominent with the increase in source and chemical reaction parameter. The thermal boundary layer thickness increases with the increase of source, chemical reaction parameter, plate surface convective heat parameter, and Schmidt number while the mass flux boundary layer thickness decreases. Moreover, the thermal boundary layer thickness, the mass boundary layer, and velocity decrease as the Prandtl number increases.

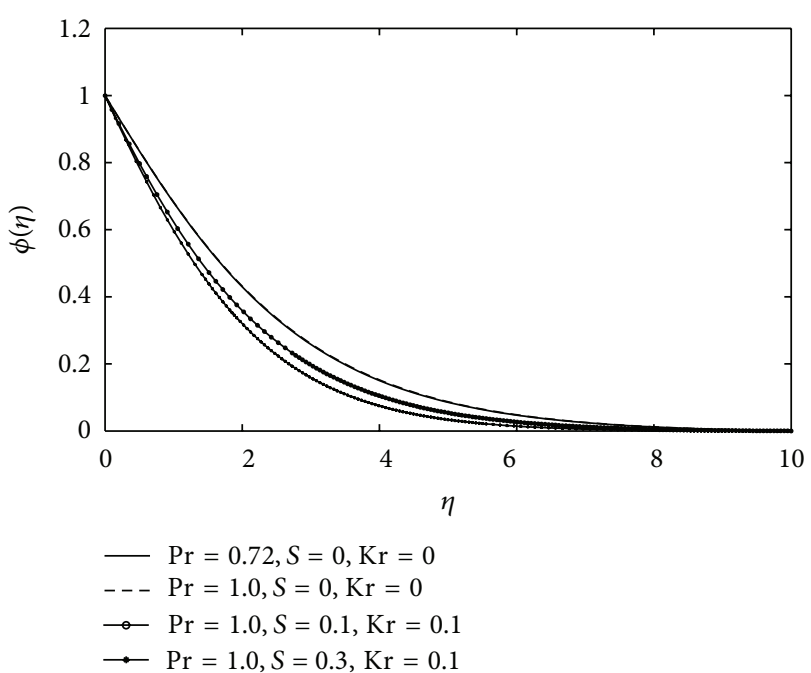

FIgURE 19: Concentration profiles for different values of $\operatorname{Pr}, S$, and $\mathrm{Kr}$ for $\mathrm{Ha}=0.1, \mathrm{Gr}=0.1, \mathrm{Gc}=0.1, \mathrm{Sc}=0.62, \mathrm{Bi}=0.1, \mathrm{Nc}=0.01$.

\section{Nomenclature}

$u, v$ : Velocity components along $x$ - and $y$-axis direction, respectively

g: Acceleration due to gravity

$Q_{0}$ : Heat source parameter

$\mu$ : Dynamic viscosity

$\nu$ : Kinematic viscosity

$U_{0}$ : Characteristic velocity at the plate

$h_{f}$ : Heat transfer coefficient

$T_{f}$ : Temperature of hot fluid at the wall

$C_{w}$ : Plate surface concentration

$C_{\infty}$ : Free stream concentration

$\beta_{c}$ : Concentration expansion coefficient

Pr: Prandtl number

D: Mass diffusivity

$\alpha$ : Thermal diffusivity

$T$ : Temperature

$C$ : Concentration

$B_{0}$ : Magnetic field strength

$\mathrm{Ha}_{x}$ : Local magnetic field parameter

$\mathrm{Bi}_{x}$ : Local convective heat transfer parameter

$T_{\infty}$ : Temperature of the fluid away from the plate

$\mathrm{Gr}_{x}$ : Local thermal Grashof number

$\mathrm{Gc}_{x}$ : Local modified Grashof number

$\mathrm{Kr}_{x}$ : Local chemical reaction parameter

$S_{x}$ : Local heat source parameter

$N_{c}$ : Concentration difference parameter

$\eta$ : $\quad$ Similarity variable

$\rho: \quad$ Fluid density

$\sigma: \quad$ Fluid electrical conductivity

$f$ : Dimensionless velocity

$\theta: \quad$ Dimensionless temperature

$\phi$ : Dimensionless concentration. 


\section{References}

[1] K. Vajrevelu and J. Nayfeh, "Hydromagnetic convection at a cone and a wedge," International Communications in Heat and Mass Transfer, vol. 19, pp. 701-710, 1992.

[2] A. J. Chamkha, "Hydromagnetic three-dimensional free convection on a vertical stretching surface with heat generation or absorption," International Journal of Heat and Fluid Flow, vol. 20, no. 1, pp. 84-92, 1999.

[3] D. Moalem, "Steady state heat transfer within porous medium with temperature dependent heat generation," International Journal of Heat and Mass Transfer, vol. 19, no. 5, pp. 529-537, 1976.

[4] M. M. Rahman and M. A. Sattar, "Magnetohydrodynamic convective flow of a micropolar fluid past a continuously moving vertical porous plate in the presence of heat generation/absorption," Journal of Heat Transfer, vol. 128, no. 2, pp. 142-152, 2006.

[5] U. N. Das, R. Deka, and V. M. Soundalgekar, "Effects of mass transfer on flow past an impulsively started infinite vertical plate with constant heat flux and chemical reaction," Forschung im Ingenieurwesen/Engineering Research, vol. 60, no. 10, pp. 284287, 1994.

[6] S. P. Anjalidevi and R. Kandasamy, "Effects of chemical reaction, heat and mass transfer on laminar flow along a semi infinite horizontal plate," Heat and Mass Transfer, vol. 35, no. 6, pp. 465467, 1999.

[7] M. A. Seddeek, A. A. Darwish, and M. S. Abdelmeguid, "Effects of chemical reaction and variable viscosity on hydromagnetic mixed convection heat and mass transfer for Hiemenz flow through porous media with radiation," Communications in Nonlinear Science and Numerical Simulation, vol. 12, no. 2, pp. 195-213, 2007.

[8] P. M. Patil and P. S. Kulkarni, "Effects of chemical reaction on free convective flow of a polar fluid through a porous medium in the presence of internal heat generation," International Journal of Thermal Sciences, vol. 47, no. 8, pp. 1043-1054, 2008.

[9] A. M. Salem and M. Abd El-Aziz, "Effect of Hall currents and chemical reaction on hydromagnetic flow of a stretching vertical surface with internal heat generation/absorption," Applied Mathematical Modelling, vol. 32, no. 7, pp. 1236-1254, 2008.

[10] F. S. Ibrahim, A. M. Elaiw, and A. A. Bakr, "Effect of the chemical reaction and radiation absorption on the unsteady MHD free convection flow past a semi infinite vertical permeable moving plate with heat source and suction," Communications in Nonlinear Science and Numerical Simulation, vol. 13, no. 6, pp. 1056-1066, 2008.

[11] S. K. Parida, M. Acharya, G. C. Dash, and S. Panda, "MHD heat and mass transfer in a rotating system with periodic suction," Arabian Journal for Science and Engineering, vol. 36, no. 6, pp. 1139-1151, 2011.

[12] R. Rajeswari, B. Jothiram, and V. K. Nelson, "Chemical reaction, heat and mass transfer on nonlinear MHD boundary layer flow through a vertical porous surface in the presence of suction," Applied Mathematical Sciences, vol. 3, no. 49-52, pp. 2469-2480, 2009.

[13] A. Mahdy, "Effect of chemical reaction and heat generation or absorption on double-diffusive convection from a vertical truncated cone in porous media with variable viscosity," International Communications in Heat and Mass Transfer, vol. 37, no. 5, pp. 548-554, 2010.
[14] D. Pal and B. Talukdar, "Perturbation analysis of unsteady magnetohydrodynamic convective heat and mass transfer in a boundary layer slip flow past a vertical permeable plate with thermal radiation and chemical reaction," Communications in Nonlinear Science and Numerical Simulation, vol. 15, no. 7, pp. 1813-1830, 2010.

[15] O. D. Makinde and A. Ogulu, "The effect of thermal radiation on the heat and mass transfer flow of a variable viscosity fluid past a vertical porous plate permeated by a transverse magnetic field," Chemical Engineering Communications, vol. 195, no. 12, pp. 1575-1584, 2008.

[16] O. D. Makinde, "MHD mixed-convection interaction with thermal radiation and nth order chemical reaction past a vertical porous plate embedded in a porous medium," Chemical Engineering Communications, vol. 198, no. 4, pp. 590-608, 2011.

[17] A. Aziz, "A similarity solution for laminar thermal boundary layer over a flat plate with a convective surface boundary condition," Communications in Nonlinear Science and Numerical Simulation, vol. 14, no. 4, pp. 1064-1068, 2009.

[18] P. O. Olanrewaju, O. T. Arulogun, and K. Adebimpe, "Internal heat generation effect on thermal boundary layer with a convective surface boundary condition," American Journal of Fluid Dynamics, vol. 2, no. 1, pp. 1-4, 2012.

[19] O. D. Makinde, "On MHD heat and mass transfer over a moving vertical plate with a convective surface boundary condition," Canadian Journal of Chemical Engineering, vol. 88, no. 6, pp. 983-990, 2010.

[20] O. D. Makinde, "Similarity solution of hydromagnetic heat and mass transfer over a vertical plate with a convective surface boundary condition," International Journal of Physical Sciences, vol. 5, no. 6, pp. 700-710, 2010.

[21] O. D. Makinde, "Similarity solution for natural convection from a moving vertical plate with internal heat generation and a convective boundary condition," Thermal Science, vol. 15, supplement 1, pp. S137-S143, 2011.

[22] K. Gangadhar, N. B. Reddy, and P. K. Kameswaran, "Similarity solution of hydromagnetic heat and mass transfer over a vertical plate with convective surface boundary condition and chemical reaction," International Journal of Nonlinear Science, vol. 3, no. 3, pp. 298-307, 2012.

[23] N.F.M. Noor, S. Abbabandy, and I. Hasim, "Heat and Mass Transfer of thermophoretic MHD flow over an inclined radiate isothermal permeable surface in presence of heat source /sink," International Journal of Heat and Mass Transfer, vol. 55, no. 7, pp. 2122-2128, 2012.

[24] V. Bisht, M. Kumar, and Z. Uddin, "Effect of variable thermal conductivity and chemical reaction on steady mixed convection boundary layer flow with heat and mass transfer inside a cone due to a point," Journal of Applied Fluid Mechanics, vol. 4, no. 4, pp. 59-63, 2011.

[25] A. A. Bakr, "Effects of chemical reaction on MHD free convection and mass transfer flow of a micropolar fluid with oscillatory plate velocity and constant heat source in a rotating frame of reference," Communications in Nonlinear Science and Numerical Simulation, vol. 16, no. 2, pp. 698-710, 2011. 

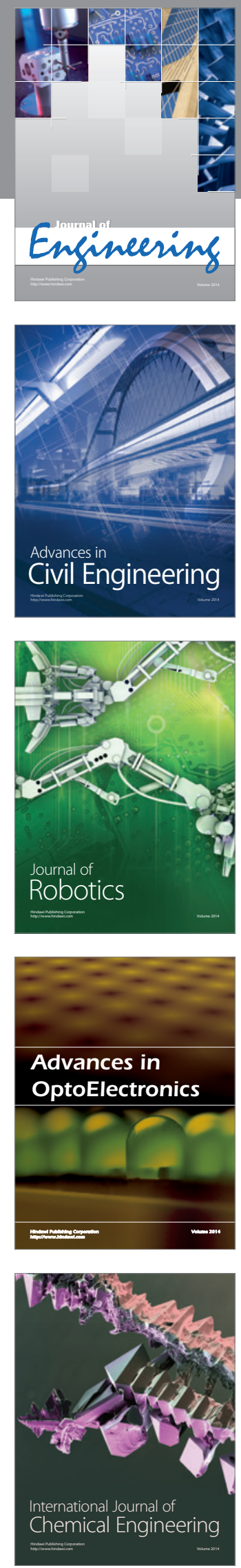

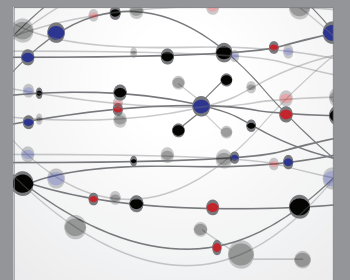

The Scientific World Journal
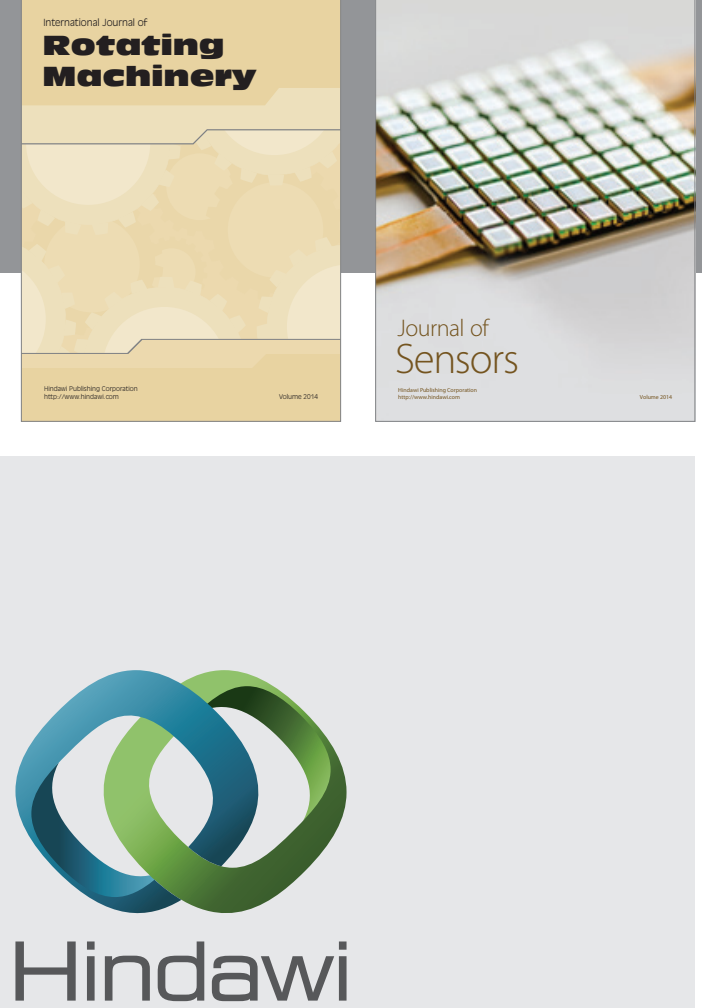

Submit your manuscripts at http://www.hindawi.com
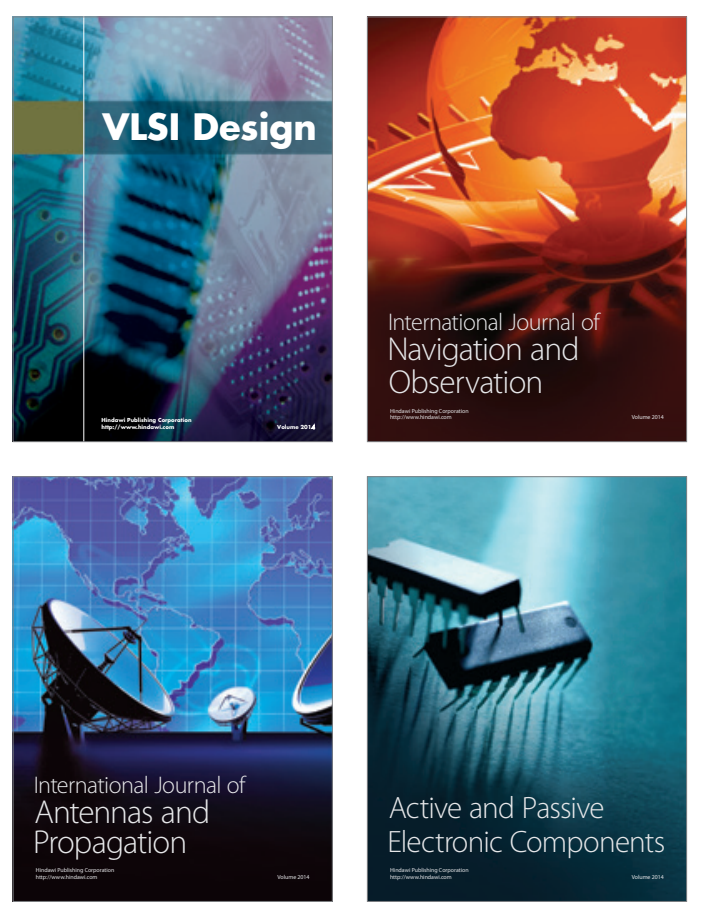
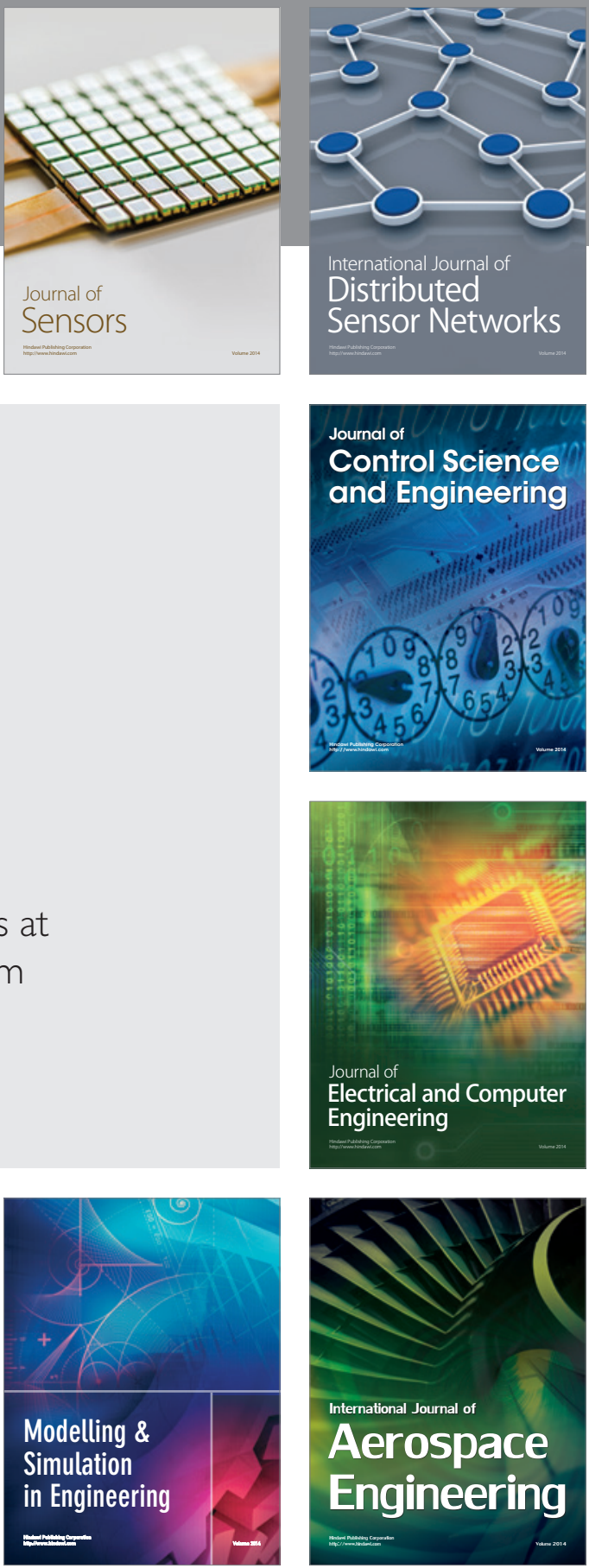

Journal of

Control Science

and Engineering
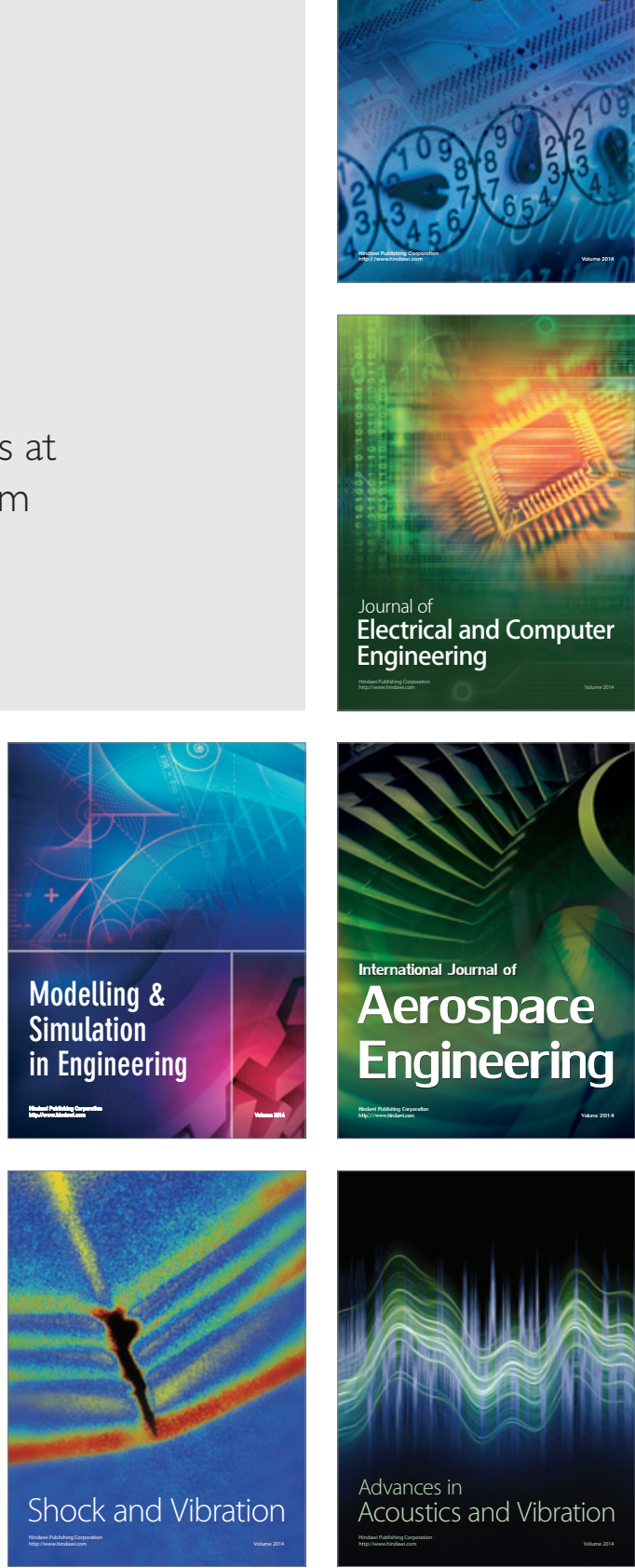